\title{
Synthesis and thermophysical studies of polyanilines
}

\author{
K S PATIL ${ }^{1}$, P H ZOPE ${ }^{1}$, U T PATIL ${ }^{1}$, P D PATIL ${ }^{2}$, R S DUBEY ${ }^{3}$ and G R GUPTA, R $^{4, *}$ \\ ${ }^{1}$ SSTs College of Engineering and Technology, Bambhori, Jalgaon 425001, India \\ ${ }^{2}$ School of Chemical Sciences, North Maharashtra University, Jalgaon 425001, India \\ ${ }^{3}$ Swarnandhra College of Engineering and Technology, Narsapur 534275, India \\ ${ }^{4}$ Institute of Chemical Technology, Matunga, Mumbai 400019, India \\ *Author for correspondence (grgupta83@ gmail.com)
}

MS received 7 September 2017; accepted 28 May 2018; published online 30 January 2019

\begin{abstract}
Aniline was polymerized under different experimental conditions such as interfacial polymerization, rapid mixing in hydrochloric acid medium and classical bulk polymerization method using $p$-toluene sulphonic acid. The resulting polyanilines were characterized by infrared, X-ray diffraction, conductance and scanning electron microscopic analysis. The main emphasis of the paper is to study the thermal response of the synthesized polyanilines by thermogravimetric analysis and differential scanning calorimetry (DSC). The DSC data were utilized further to calculate the specific heat capacities of the synthesized polyanilines as a function of temperature.
\end{abstract}

Keywords. Conducting polymers; polyanilines; specific heat capacity.

\section{Introduction}

Polyaniline is one of the most extensively studied conducting polymers because of its simple synthesis and doping/dedoping chemistry [1,2], comparatively low cost, moderate-to-high conductivity and excellent moisture and air stability. Polyanilines have a broad scope of applications in rechargeable batteries, modern optical information storage, shielding of electromagnetic interference [3-5], microwave and radar absorbing materials [6], sensors, indicators, catalysts [7-9], electronic and bioelectronic components [10], membranes [11], electrochemical capacitors [12], electrochromic devices, nonlinear optical and light-emitting devices [13-15], electromechanical actuators [16], antistatic [17], anti-corrosion coatings [18] etc. Their electromagnetic and optical properties are strongly influenced by their oxidation state and degree of protonation [19-21]. Therefore, researchers have shown great attention to synthesize polyanilines by various methods including chemical as well as electrochemical oxidation processes [22].

Similarly, thermal methods of analysis are the most important tools in the arsenal of analytical chemistry, and are useful to extract structure-related information of the materials which are under investigation. Probably the most used techniques amongst the thermal analysis are thermogravimetric analysis (TGA) and differential scanning calorimetry (DSC). TGA is used in all types of applications, providing information about the bonding of components within the sample. On the other hand, DSC is used to characterize the physical properties (specific heat capacity, \% crystallinity etc.) of the materials and how they change with respect to temperature [23]. Furthermore, these methods have extensively been used to extract subtle information about the materials, namely, kinetics of reactions, mechanisms, and quality control of raw materials, rheological changes and most importantly the glass transitions of the materials [24].

Herein, the attempts have been made to characterize the synthesized polyanilines by Fourier transform infrared (FT-IR), X-ray diffraction (XRD), scanning electron microscope (SEM) and TGA-DSC techniques. In the paper, the thermal data obtained by DSC have been processed to determine the specific heat capacity $\left(C_{\mathrm{p}}\right)$ as a function of temperature for the synthesized polyanilines. All these results are presented and discussed in the following sections.

\section{Materials and methods}

\subsection{Materials}

Reagents required were procured from Sigma-Aldrich and SD Fine. Aniline was first distilled before use. Catalyst, hydrochloric acid $(\mathrm{HCl}, 35 \%$ by weight in water), $p$-toluene sulphonic acid and oxidant ammonium persulphate were used as received. Water used was quartz doubly distilled.

\subsection{Synthesis of polyanilines}

Polyanilines were synthesized in different ways. In a first method doping with strong $\mathrm{HCl}$ was used while in another 
method $p$-toluene sulphonic acid was used. The details are given below.

\subsection{Route 1: HCl-doped polyaniline (interfacial polymerization; sample A)}

Interfacial polymerization is well developed to obtain polyaniline nanofibre material. A solution of aniline $(0.3 \mathrm{~mol})$ was mixed with chloroform and subsequently mixed with $0.08 \mathrm{~mol}$ solution of ammonium persulphate in $\mathrm{HCl}$. This mixing corresponds to a 1:1 stoichiometry of the reactant aniline persulphate. The mixed solution turns into a two-phase system having an emulsion type interface (similar to that of an oil and water interface), this is attributed to the limited solubility of chloroform in water. The oxidation and polymerization reactions yielded green polyaniline nanofibres. The resulting nanofibres were washed with $1 \mathrm{~N} \mathrm{HCl}$ in order to remove unreacted aniline followed by the treatment of distilled water to remove unreacted ammonium persulphate and finally washed with acetone to remove any organic impurities if present. Thereafter, the filtered material was dried and stored in a desiccator [25].

\subsection{Route 2: HCl-doped polyaniline (rapid mixing method; sample $B$ )}

A rapid mixing method was developed to obtain polyaniline nanofibres. A $0.32 \mathrm{M}$ solution of aniline in water was mixed with a $0.08 \mathrm{M}$ solution of ammonium persulphate in $\mathrm{HCl}$ with a 1:1 stoichiometry. The two solutions were mixed with vigorous stirring on a magnetic stirrer (800 rpm). The aniline monomer completely reacted with acid solution and needle-like fibre structures were formed. The obtained green polyanilines were treated with $1 \mathrm{~N} \mathrm{HCl}$, distilled water and acetone to remove unreacted aniline ammonium persulphate and any organic impurities, respectively. The resulting polyanilines were dried and stored in a desiccator [25].

\subsection{Route 3: p-toluene sulphonic acid-doped polyaniline (bulk polymerization; sample $C$ )}

Pure polyaniline particles were synthesized using chemical polymerization. A $500 \mathrm{ml}$ conical flask was charged with a solution of $p$-toluene sulphonic acid:ammonium persulphate at a fixed ratio of $30 \mathrm{mmol}: 18 \mathrm{mmol}$ in $200 \mathrm{ml}$ deionized water. The solution was subjected to 1-h sonication in an icewater bath. Thereafter, the solution of aniline $(36 \mathrm{mmol}$ in $50 \mathrm{ml}$ deionized water, molar ratio of ammonium persulphate: $p$-toluene sulphonic acid:aniline $=3: 5: 6$ ) was added in a portion and the whole reaction mixture was sonicated for an hour in the ice-water bath for polymerization. The resulting polyaniline was filtered and collected after several water washings. The obtained polyaniline powder was dried and stored in a desiccator [26].

\subsection{Analytical method used for characterization of the synthesized polyanilines}

2.6a FT-IR studies of polyanilines: FT-IR analysis was performed on a Bruker alpha Eco-ATR. The peaks for the polyaniline base and their salts appear in the same region and with similar intensities except for having marginal differences. The spectrum is shown in figure 1 (1a and $b$ for polyanilines doped with $\mathrm{HCl}$, while 1c for polyanilines doped with $p$-toluene sulphonic acid) and the analysis reveals that the main characteristic absorption peaks of polyaniline samples are appearing at 3450-3430, 2500-2250, 1500-1450, 1100-1020, 750 and $650 \mathrm{~cm}^{-1}$, respectively. The peak at $3450-3430 \mathrm{~cm}^{-1}$ is attributed to the stretching mode of the $\mathrm{N}-\mathrm{H}$ band, while the $\mathrm{C}-\mathrm{H} \mathrm{sp}{ }^{3}$ stretch and $\mathrm{C}=\mathrm{C}$ stretch mode for the benzenoid group appear at 2500-2250 and 1500-1450 $\mathrm{cm}^{-1}$, respectively. The peak observed at $1100-1020 \mathrm{~cm}^{-1}$ can be attributed to the quinonoid unit of polyaniline [27]. The peaks at 750 and $650 \mathrm{~cm}^{-1}$ represent the $\mathrm{C}-\mathrm{H}$ and $\mathrm{C}-\mathrm{C}$ bands of the benzenoid group.

2.6b Microscopic morphology of polyanilines: SEM analysis of polyaniline samples was performed on Hitachi-S4800 (type-II) instrument, Japan. As shown in figure $2 \mathrm{a}$ and $b$, the surface morphologies of the polyaniline$\mathrm{HCl}$ exhibit uniformly grown polyaniline particles as well as their interlocking between some nanofibre-like materials. The SEM photograph shown in figure $2 \mathrm{c}$ indicates that the particles in polyaniline- $p$-toluene sulphonic acid are in the form of agglomer whereas polyaniline$\mathrm{HCl}$ nanofibres are non-uniform in their size distribution, porous and voids as seen in figure $2 \mathrm{a}$ and $\mathrm{b}[21,28]$. The diameters of the nanofibres are estimated to be about $50-60 \mathrm{~nm}$ and the length of the nanofibres is about 100$950 \mathrm{~nm}$.

2.6c XRD spectrum analysis of polyanilines: To investigate the structural characteristics, we obtained an XRD pattern of the studied samples in powder form. The diffractograms were obtained with Bruker-D8-Advanced instrument, Germany. The wavelength (of source) corresponds to $(\mathrm{Cu}-\mathrm{K} \alpha) 1.53 \AA$. The range of the Braggs angles was between 10 and $80^{\circ}(2 \theta)$ for the samples.

The XRD patterns of polyanilines are shown in figure 3, which exhibit diffraction peaks at about $2 \theta=18^{\circ}$. The sharp peak around $18^{\circ}$ is the characteristic peak for the polyaniline materials. The X-ray data highlight that the synthesized polyaniline materials have partial crystallinity with conducting metallic islands separated by large amorphous regions. The peak around $\left(26^{\circ}\right)$ is a characteristic peak indicating that some parts of the polyaniline sub-chains become rigid and well-ordered due to interchain packing between the polycation and $p$-toluene sulphonate anion (sample $\mathrm{C}$ ) [29-31]. 

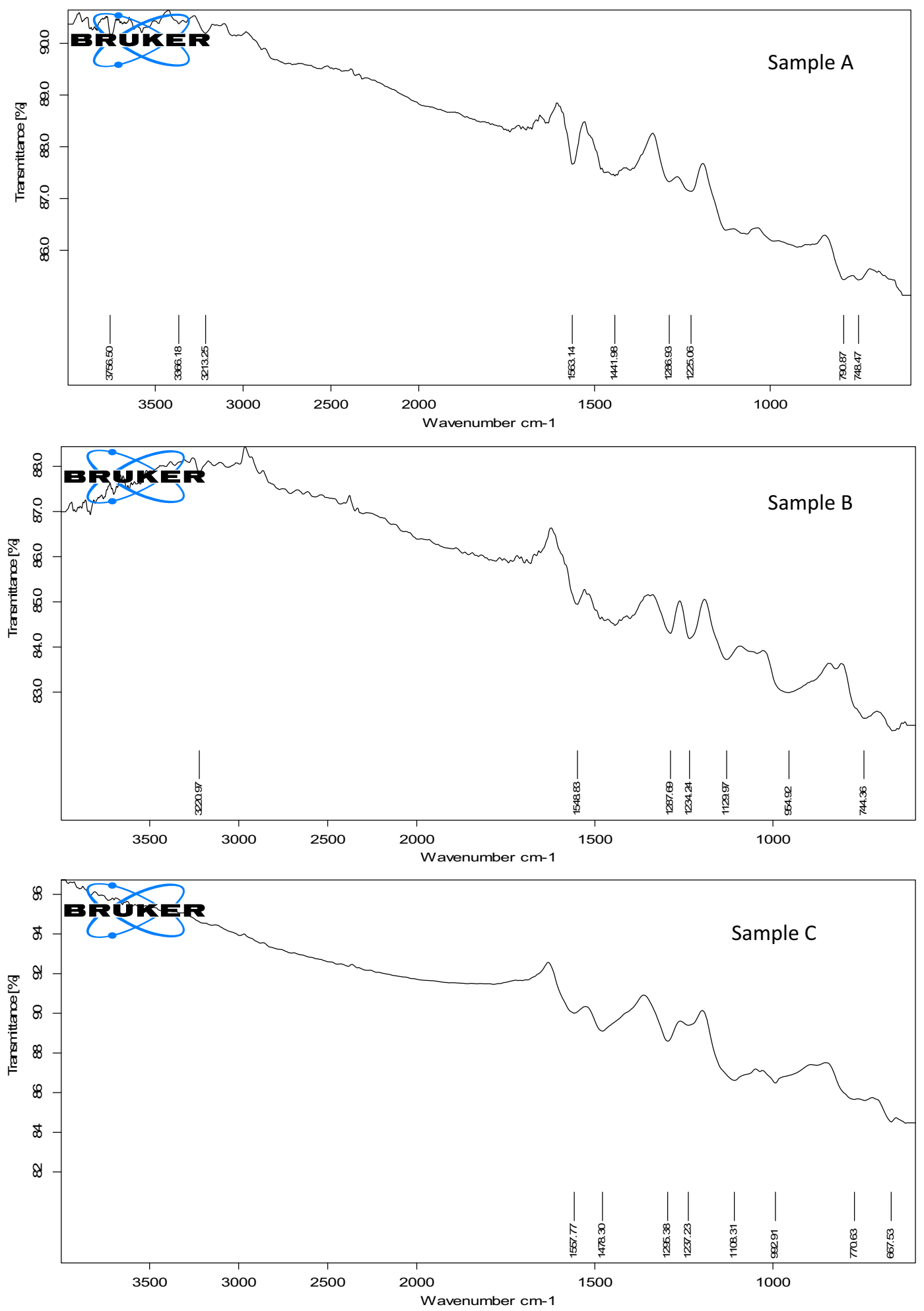

Figure 1. FT-IR spectra of polyanilines.

2.6d Thermal analysis of polyanilines: The TGA-DSC analysis of the synthesized polyaniline materials was performed on the TA instrument (model: SDT Q $600 \mathrm{~V}$ 20.9 ) with a temperature accuracy of $\pm 0.5 \mathrm{~K}$ under a $\mathrm{N}_{2}$ atmosphere, which was placed in an air-conditioned lab having humidity of 5-10\%.

Samples having weights in the range of $4-10 \mathrm{mg}$ were used for the measurements. The sample volumes of the alumina 

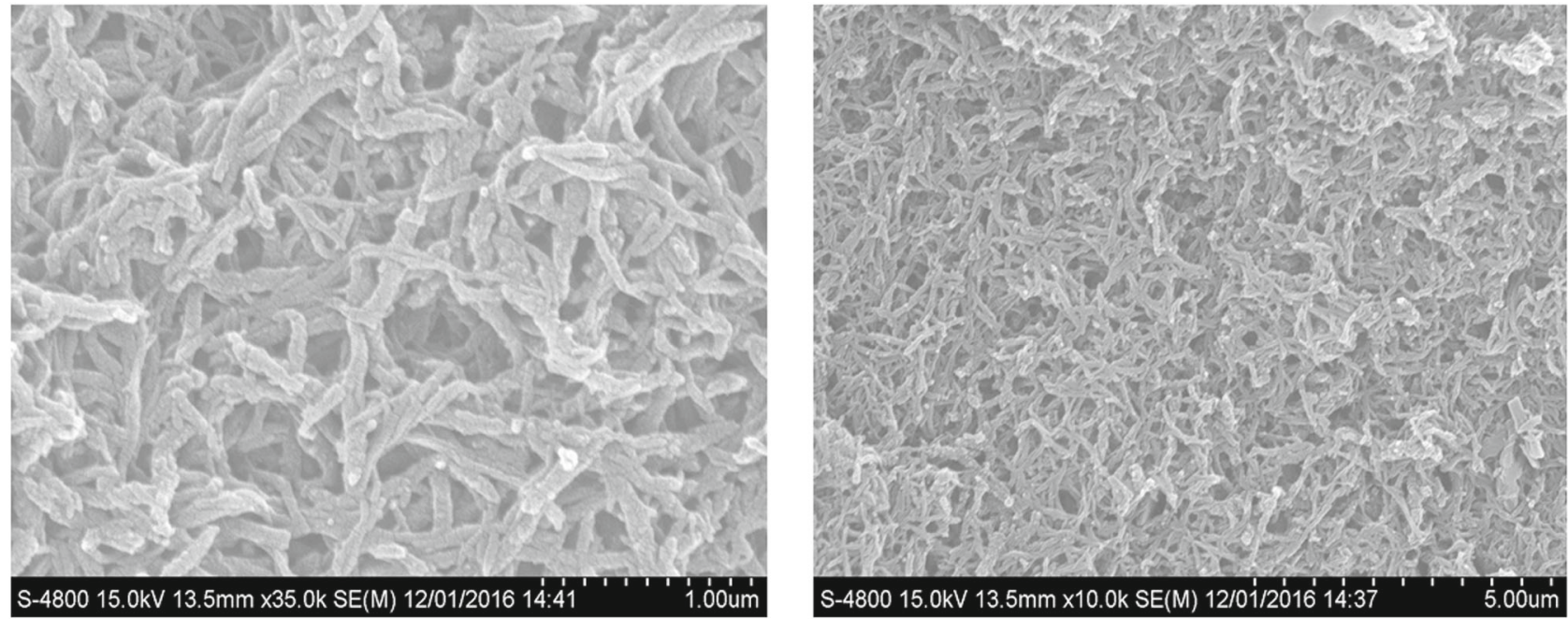

a (Sample A)

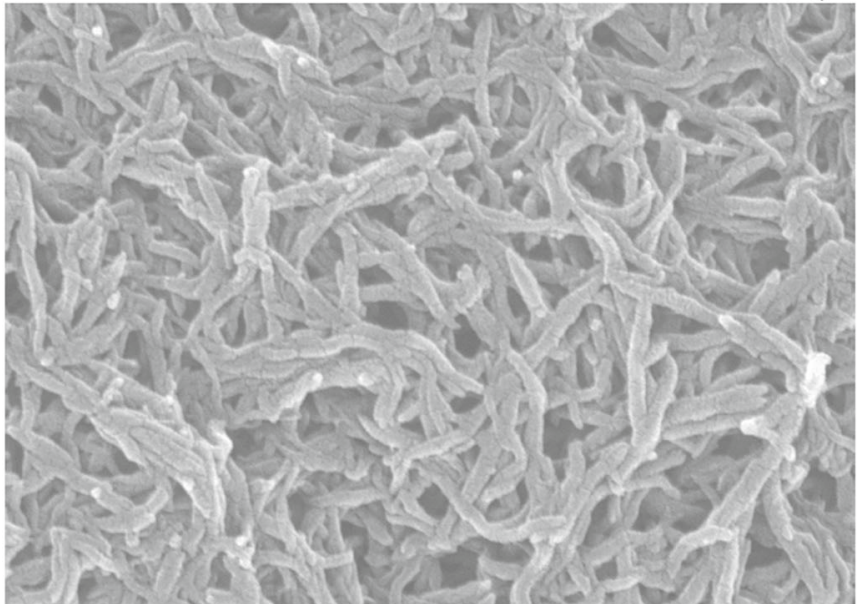

S-4800 15.0kV 13.7mm x40.0k SE(M) 12/01/2016 14:49

'1.00um S-4800 15.0kV 13.7mm x11.0k SE(M) 12/01/2016 14:45

5.00 um

b ( Sample B)

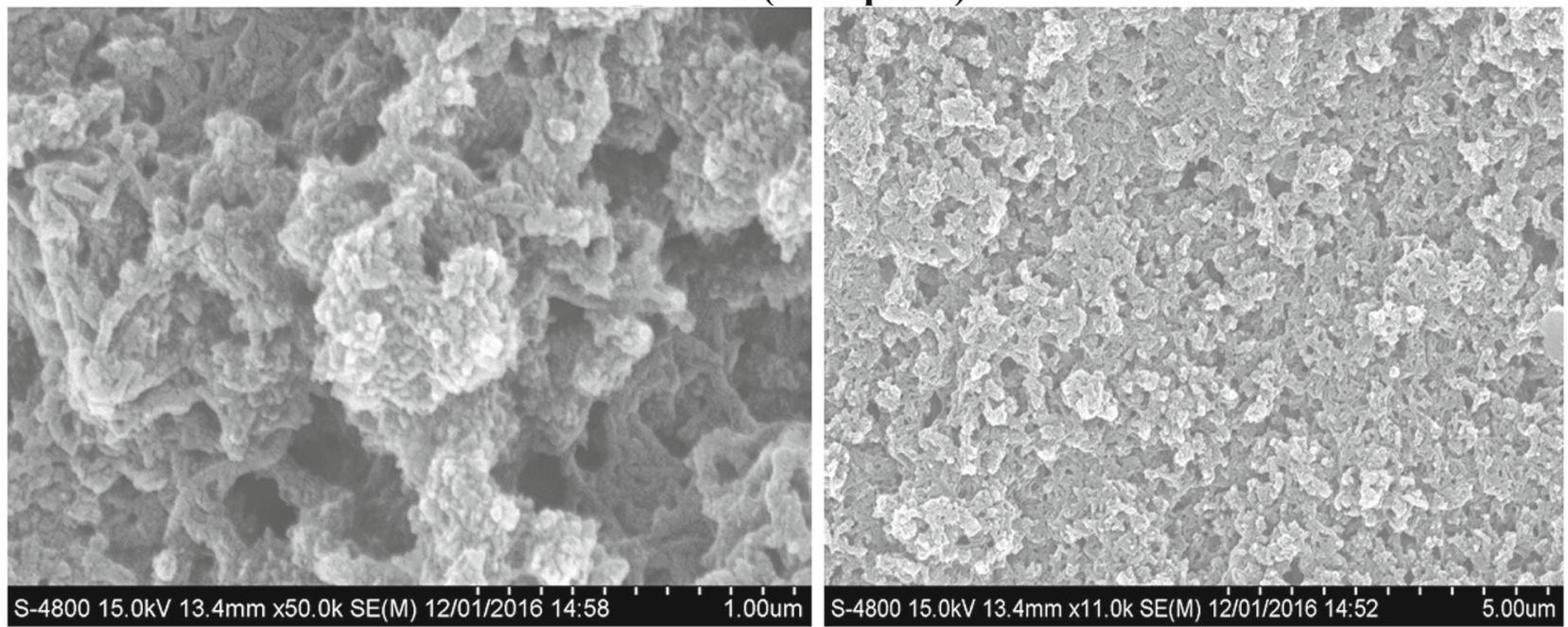

c (Sample C)

Figure 2. SEM images of the synthesized polyanilines. 


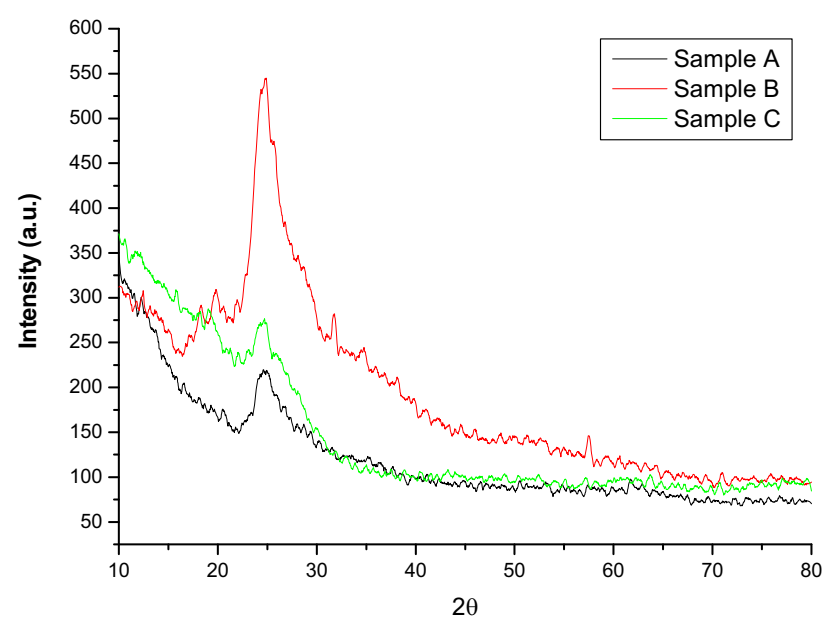

Figure 3. X-ray diffraction patterns of polyanilines.

pans were $10 \mathrm{~mm}^{3}$ with a cell volume of $3.4 \mathrm{ml}$ and were subjected to nitrogen purging at a flow rate of $50 \mathrm{ml} \mathrm{min}^{-1}$. The heating rate of the sample was always maintained at $5 \mathrm{~K} \mathrm{~min}^{-1}$. The instrument was calibrated for differential temperature using an empty pan, sapphire and fusion point of $\mathrm{Zn}$, while heat flow measurements were performed in the range of 313.15-1073.15 K. No sample base line has been used to correct the effects of the reference side, by which a high-quality linear straight line was obtained for the parameters: uncalibrated temperature difference and actual temperature. Similar results were obtained for sapphire $(59.719 \mathrm{mg})$.

Thus, after establishing suitable working conditions for the instrument and setting up proper conditions, we subjected our $\mathrm{KCl}$ samples as a standard, samples $\mathrm{A}, \mathrm{B}$ and $\mathrm{C}$ of the polyaniline materials for obtaining thermograms and heat flow measurements by DSC over the temperature range of 298.15-773.15 K.

The details of the calculations of sample deflections with appropriate corrections are given below. A systematic and efficient computing was adopted to calculate specific heat capacity for solids using DSC-SDT-Q-600 instrument.

(A) Performance of thermistors was checked for a selected temperature range and obtained the signal in $\mu \mathrm{V}$.

(B) The calibrated weight analysis was performed for a selected temperature range and data were obtained in $\mu \mathrm{V} \mathrm{mg}{ }^{-1}$.

(C) An empty pan signal was studied and its contribution (in $\mu \mathrm{V}$ ) at different temperatures was obtained.

(D) Sapphire was studied as a calibrant standard and its DSC profile was obtained in a temperature range of $55-1184^{\circ} \mathrm{C}(328.15-1457.15 \mathrm{~K})$. The corresponding signal was obtained in terms of $\mu \mathrm{V} \mathrm{mg}^{-1}$.

(E) The correction for pan signal data was applied using appropriate corrections due to matching of thermistors.
(F) The signal of weights was subtracted from the signal of sapphire. The resulting signal was multiplied by the weight of sapphire and finally converted the emf into volts $(\mathrm{V})$. Then, the pan signal in volts was subtracted from the sapphire signal in volts.

(G) Zinc metal was used to assess the temperature difference, i.e., $\mathrm{d} T\left({ }^{\circ} \mathrm{C}\right)$ between two pans. A DSC scan of the zinc metal was obtained in a temperature range of $25-490^{\circ} \mathrm{C}(298.15-763.15 \mathrm{~K})$. The zinc melts at $425.84^{\circ} \mathrm{C}(698.99 \mathrm{~K})$. The area under the endothermic curve was calculated and was further used to obtain heat of fusion of the zinc metal as $107.1 \mathrm{~J} \mathrm{~g}^{-1}$. The data are in excellent agreement with the literature data.

(H) From the specific heat capacity values for sapphire and the measured $\mathrm{d} T$ values at different temperatures, the enthalpy change for sapphire as a function of temperature was calculated in $\mathrm{J} \mathrm{s}^{-1}$. The data yielded the values of current $(I)$ generated via the use of equation $\Delta H=I^{2} R t$. Using the average of current values, signal values for sapphire were converted into power (watts) values at the studied temperatures. From the measured current, the DSC signals for pan and sapphire appropriately converted into the power unit of watts. The appropriate amplitudes for sapphire and pan were obtained and a pan correction for amplitude data of sapphire was applied. Similar corrections for samples of studied ionic solids were made in respect to DSC signal amplitude in watts.

(I) The amplitude ratios for salt/sapphire (corrected) as well as the weight ratio (sapphire/salt) were calculated and further used to obtain specific heat capacity at constant pressure $\left(C_{\mathrm{p}}\right)$ as a function of temperature for the studied salts, using the below equation:

$$
K \cdot C_{\mathrm{p}}=\frac{\text { Amplitude ratio } \times \text { weight ratio }}{\text { scanning rate }}
$$

where $K$ is the sensitivity in $\mathrm{J}^{-1} \mathrm{~g} \mathrm{~s}$ which has been taken as unity (i.e., 1). The above methodology was applied to the data of $\mathrm{KCl}$. The calculated values of specific heat capacity for $\mathrm{KCl}$ show excellent agreement above $65^{\circ} \mathrm{C}(348.15 \mathrm{~K})$ up to $345^{\circ} \mathrm{C}(618.15 \mathrm{~K})$. We observed that sapphire as a standard for specific heat capacity measurements yielded reliable results above $65^{\circ} \mathrm{C}(348.15 \mathrm{~K})$, as there are structural transitions reported at about $40-50^{\circ} \mathrm{C}(313.15-323.15 \mathrm{~K})$ for sapphire.

Based on this methodology, the specific heat value calculations at different temperatures were calculated using equation (1). The accuracy of the instrument was verified by determining the specific heat capacity of $\mathrm{KCl}$. The specific heat capacity values as a function of temperature for $\mathrm{KCl}$ are plotted for the salt in figure 4 . The estimated accuracy of the measured values is $\pm 2 \%$ over this temperature 


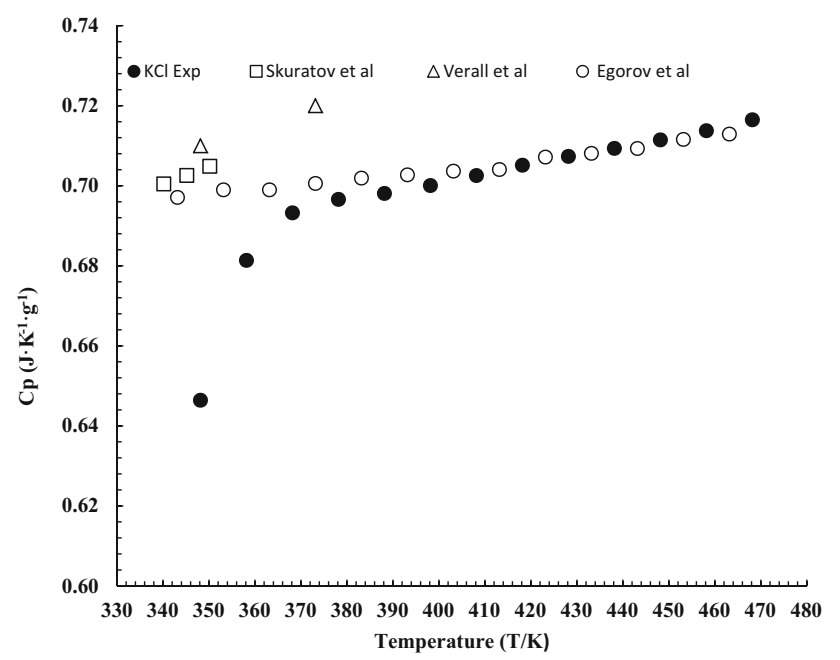

Figure 4. Comparison of specific heat capacity data for potassium

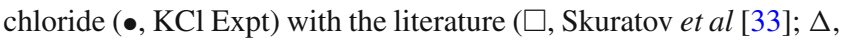
Verall et al [32]; o, Egorov et al [33]).

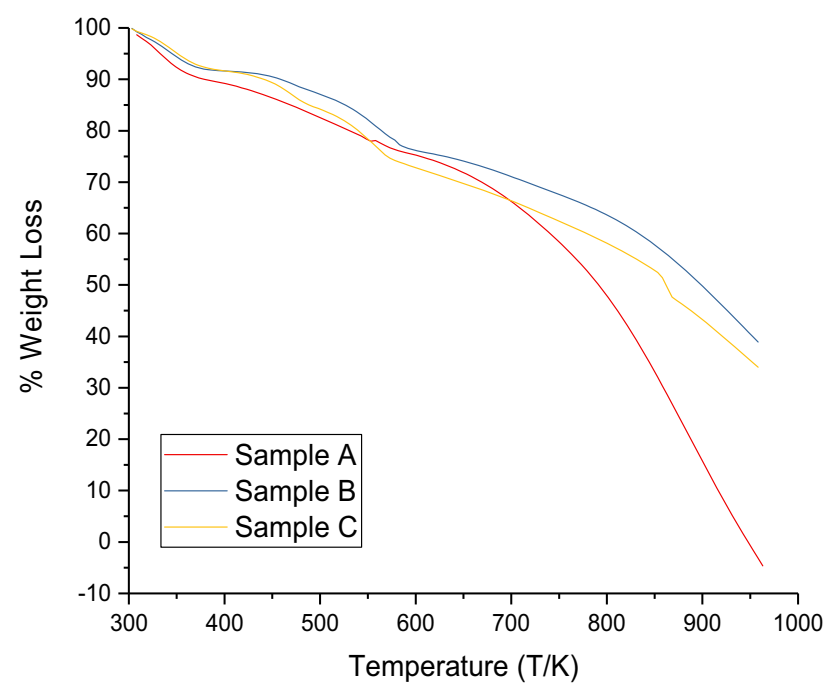

Figure 5. TGA of polyanilines.

range, when the data were compared with the literature data $[32,33]$.

\section{Results and discussion}

\subsection{TGA-DTG of polyanilines}

Thermal analysis of the synthesized polyanilines reveals an organic metal, i.e., polyanilines are thermally reactive materials, as shown in figure 5. The thermal response of both samples is quite similar and they change continually and cause thermal-induced degradation.

Derivative thermogravimetry of the synthesized polyaniline samples in figure 6 enlightens that samples B and C are

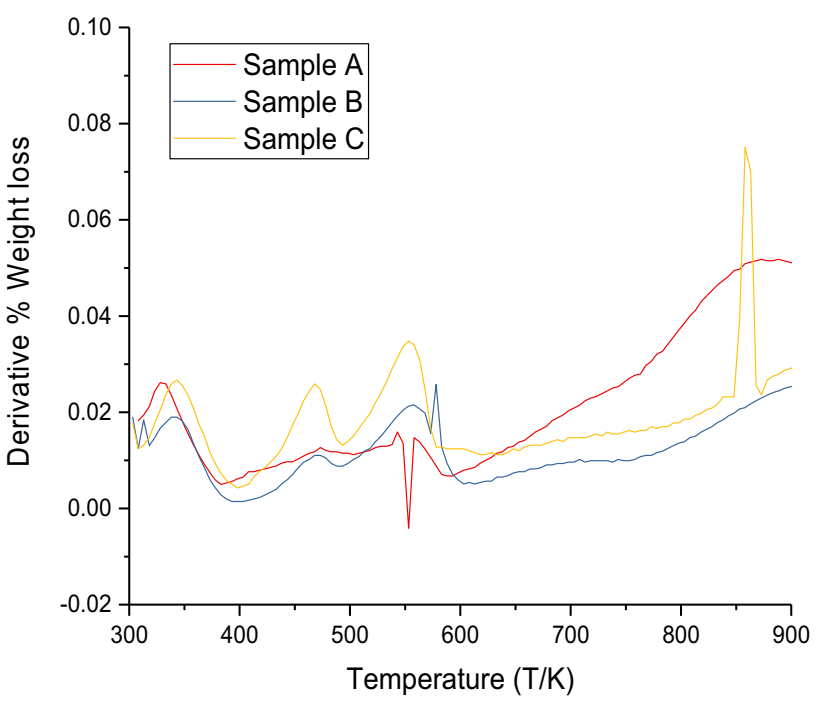

Figure 6. DTG of polyanilines.

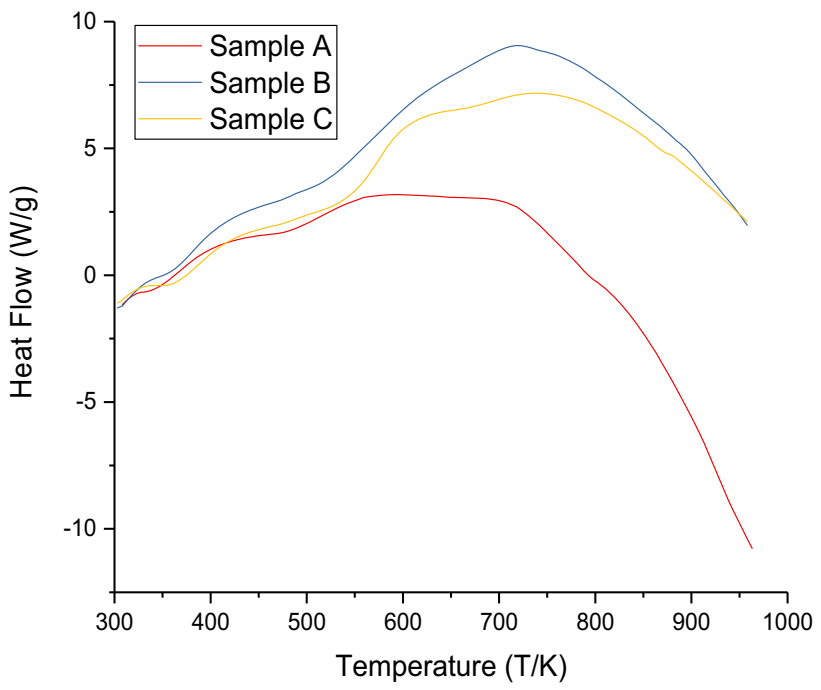

Figure 7. DSC of polyanilines.

structurally very similar and show a quite similar trend of DTG, while sample A of the polyaniline is different from samples B and C. We believe that the difference arises initially due to the removal of residual impurities absorbed and due to structural differences between the samples of polyaniline. The structural differences may arise because of packing and other structural differences due to different methods used for the synthesis. The details need to be investigated further.

\subsection{DSC analysis of polyanilines}

The utility of DSC-TGA techniques in the materials science is due to the uniqueness of the techniques which seizes attention of the scientific community [34]. TGA-DTG analysis of 
Table 1. Specific heat capacity $\left(C_{\mathrm{p}}\right)$ data of the samples of polyanilines.

\begin{tabular}{|c|c|c|c|}
\hline $\begin{array}{l}\text { Temperature } \\
T(\mathrm{~K})\end{array}$ & $\begin{array}{c}\text { Sample A } \\
\left(\mathrm{J} \mathrm{K}^{-1} \mathrm{~g}^{-1}\right)\end{array}$ & $\begin{array}{c}\text { Sample B } \\
\left(\mathrm{J} \mathrm{K}^{-1} \mathrm{~g}^{-1}\right)\end{array}$ & $\begin{array}{c}\text { Sample C } \\
\left(\mathrm{J} \mathrm{K}^{-1} \mathrm{~g}^{-1}\right)\end{array}$ \\
\hline 338.15 & 3.89 & 1.85 & 2.85 \\
\hline 348.15 & 4.07 & 2.00 & 3.04 \\
\hline 358.15 & 4.10 & 2.05 & 3.11 \\
\hline 368.15 & 4.09 & 2.05 & 3.11 \\
\hline 378.15 & 4.06 & 2.03 & 3.07 \\
\hline 388.15 & 4.04 & 2.00 & 3.03 \\
\hline 398.15 & 4.04 & 1.98 & 2.99 \\
\hline 408.15 & 4.05 & 1.96 & 2.95 \\
\hline 418.15 & 4.08 & 1.95 & 2.92 \\
\hline 428.15 & 4.11 & 1.94 & 2.91 \\
\hline 438.15 & 4.14 & 1.94 & 2.92 \\
\hline 448.15 & 4.19 & 1.95 & 2.93 \\
\hline 458.15 & 4.24 & 1.95 & 2.95 \\
\hline 468.15 & 4.29 & 1.97 & 2.99 \\
\hline 478.15 & 4.34 & 1.97 & 3.02 \\
\hline 488.15 & 4.37 & 1.98 & 3.03 \\
\hline 498.15 & 4.39 & 1.98 & 3.03 \\
\hline 508.15 & 4.40 & 1.98 & 3.03 \\
\hline 518.15 & 4.40 & 1.97 & 3.04 \\
\hline 528.15 & 4.40 & 1.96 & 3.03 \\
\hline 538.15 & 4.41 & 1.93 & 3.01 \\
\hline 548.15 & 4.42 & 1.90 & 2.96 \\
\hline 558.15 & 4.40 & 1.86 & 2.87 \\
\hline 568.15 & 4.44 & 1.82 & 2.72 \\
\hline 578.15 & 4.48 & 1.76 & 2.48 \\
\hline 588.15 & 4.51 & 1.70 & 2.23 \\
\hline 598.15 & 4.54 & 1.61 & 2.02 \\
\hline 608.15 & 4.58 & 1.52 & 1.84 \\
\hline 618.15 & 4.62 & 1.43 & 1.70 \\
\hline
\end{tabular}

the synthesized polyanilines has been less explored and yet cannot be used to explain structure-related interactions. While, the DSC profiles (figure 7) enable calculations of specific heat capacity of the polyaniline as a function of temperature and the obtained values of specific heat capacity for polyaniline are tabulated in table 1 for the temperature range of 338.15-618.15 K.

It is observed from figure 8 that the sample prepared by interfacial polymerization (sample A) exhibits higher value of specific heat capacity. While the derivative of polyaniline prepared by the rapid mixing method (sample B) also shows lower values than sample $C$, which was prepared by the bulk polymerization method. The nanofibres of polyaniline obtained by the interfacial method (sample A) and rapid mixing method (sample B) are both $\mathrm{HCl}$-doped, whereas sample $\mathrm{C}$ prepared by the bulk polymerization method is $p$ toluene sulphonic acid doped and partially particle in nature. Higher specific heat capacity values mean more energy storage capacity. Thus, the sample obtained from the rapid mixing is structurally more stable, allowing better lattice formation than the other two samples. The specific heat capacity

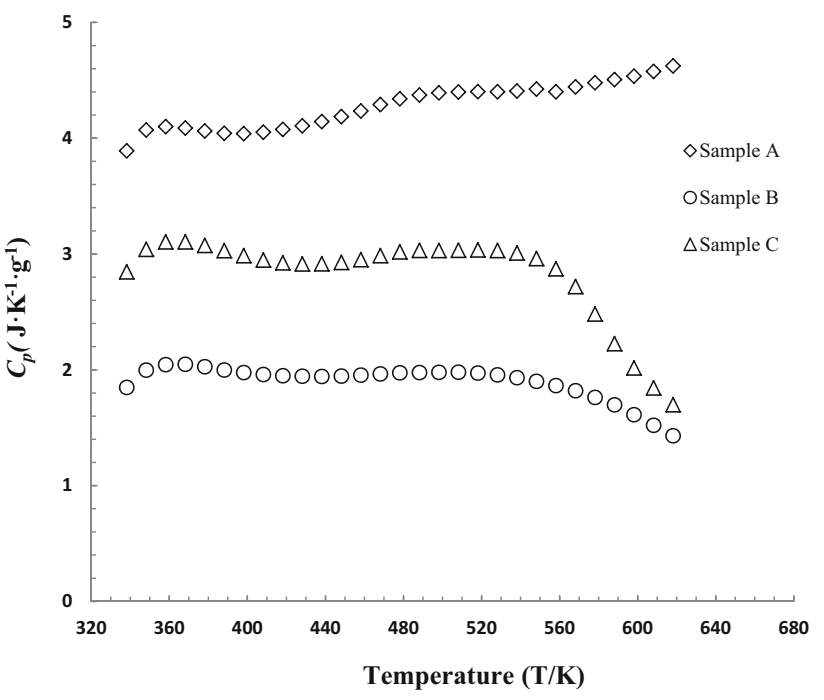

Figure 8. Specific heat capacity $\left(C_{\mathrm{p}}\right)$ of polyanilines as a function of temperature.

behaviour of the sample obtained by bulk polymerization (sample C), partially particle in nature, exhibits higher specific heat capacity values than that of the sample obtained by the rapid mixing method, hence having better structural stability at all temperatures studied. The detailed X-ray structure analysis for such sample is lacking but it seems that polyanilines can exist in different polymeric arrangements, the stability of which can be assessed on the basis of specific heat capacity data.

The thermal stability of the doped polyaniline is highly dependent on the dopant anions attached to the polymer backbone. Although, previous polyanilines doped with $p$-toluene sulphonic acid was reported to be more stable than those doped with inorganic dopants like $\mathrm{HCl}$ [35]; we observed an opposite trend in which the $\mathrm{HCl}$-doped polyaniline showed the highest thermal stability. It means that the $\mathrm{HCl}$-doped nanofibres show higher specific heat capacity value which is responsible for the high-energy storing ability as compared with the other polyaniline materials. We did not know the degree of polymerization or molecular weight of the polymers synthesized and hence refrain our self for making further comments.

These results can be interpreted in another way. The total heat capacity of the substance is made up of two parts consisting of geometrical $\left(C_{\mathrm{pgeo}}\right)$ and structural contribution, i.e., relaxational contribution given as

$$
C_{\mathrm{p} \text { Total }}=C_{\mathrm{p} \text { geo }}+C_{\text {p relaxational }}
$$

The sample of fibrous nature (sample B) seems to be accompanied by loss in relaxational contribution while samples A and $\mathrm{C}$ seem to show appreciable relaxational contribution to the values of specific heat capacity observed. From table 1, it has been observed that the glass transition temperature 
Table 2. Electrical conductivity of polyaniline- $\mathrm{HCl}$ and polyaniline- $-p$-toluene sulphonic acid.

\begin{tabular}{lc}
\hline Polyanilines & Electrical conductivity (room temperature) $\left(\mathrm{S} \mathrm{cm}^{-1}\right)$ \\
\hline Polyaniline- $\mathrm{HCl}$ (sample A) & $3.67 \times 10^{-4}$ \\
Polyaniline I-HCl (sample B) & $5.63 \times 10^{-4}$ \\
Polyaniline- $p$-toluene sulphonic acid (sample C) & $9.7 \times 10^{-2}$ \\
\hline
\end{tabular}

$\left(T_{\mathrm{g}}\right)$ appears to be around $348.15 \mathrm{~K}$; initially specific heat capacity decreases, however to establish it conclusively one needs more data points at lower temperature.

\subsection{Electrical conductivity of polyanilines}

The electrical conductivity of polyaniline pellets was measured using a Keithley 2411 A. The electrical conductivity of bulk polyaniline was observed to be 850 times than that of polyaniline nanofibres having conductivity in the range of $10^{-2}$ to $10^{-4} \mathrm{~S} \mathrm{~cm}^{-1}[27,36]$, the electrical conductivity of the fibres is not very high. This may be due to a fibrous interwoven mesh structure. The examination of table 2 reveals that the green protonated emeraldine has conductivity at a semiconductor level [24]. This value is many orders of magnitude higher than that of common polymer $10^{-9} \mathrm{~S} \mathrm{~cm}^{-1}$, but lower than that of typical metals $>10^{4} \mathrm{~S} \mathrm{~cm}^{-1}$.

Electrical conductivity of polyaniline- $p$-toluene sulphonic acid is better than polyaniline- $\mathrm{HCl}$. In polyaniline- $\mathrm{HCl}$, bipolaron is formed by the loss of two electrons from a small segment of the polymeric chain and this bipolaron easily loses protons from the $\mathrm{NH}$ groups where positive charge is localized $\oplus$

$-\stackrel{\oplus}{\mathrm{N} H}$ and forms quinoids (ES). But in polyaniline- $p$-toluene sulphonic acid, each nitrogen carries hydrogen and the bipolaron formed can easily be moved and due to this it shows better conductivity.

\section{Conclusion}

The above-mentioned polyaniline samples were obtained in the form of green polymeric material (emeraldine salt) and characterized by FT-IR, XRD and SEM techniques. The spectral data of polyanilines are in harmony with the data found in the literature. The DSC profile of the polyaniline material was utilized to obtain specific heat capacity as a function of temperature. To our knowledge, this is the first report in which an attempt was made to study the specific heat capacity parameter for conducting polymers like polyaniline using DSC. We found that specific heat capacity values increase with temperature. Since specific heat capacity is a measure of energy storage for translational, rotational and vibrational motions at degrees of freedom, the comparatively higher value obtained for the sample prepared using the rapid mixing method (sample B) shows more structural ordering than the other polyaniline samples. The electrical properties of polyanilines are in close agreement with the value found in the literature.

\section{Acknowledgements}

We gratefully thank Professor K J Patil, Ex. Professor, Shivaji University, Kolhapur for knowledge sharing and technical writing. The authors are also thankful to the management and Principal Dr K S Wani for the financial support of the project from Shram Sadhana Research Promotion Scheme No. COET/SSRPS/1626/019/13 dated 6 Sept, 2013 started in SSBTs COET Bambhori, Jalgaon (M.S.), India to increase the research activity in the institute. Also many thanks to the North Maharashtra University, Jalgaon and Shivaji University, Kolhapur for providing characterization facility.

\section{References}

[1] Stejskal J and Gilbert R G 2002 Pure Appl. Chem. 74857

[2] Chiang J C and MacDiarmid A G 1986 Synth. Met. 13193

[3] MacDiarmid A G, Yang L S, Huang W S and Humphrey B D 1987 Synth. Met. 18393

[4] McCall R P, Ginder J M, Leng J M, Coplin K A, Ye H J, Epstein A J et al 1991 Synth. Met. 411329

[5] Trivedi D C and Dhawan S K 1993 Synth. Met. 59267

[6] Makeiff D A and Huber T 2006 Synth. Met. 156497

[7] Dutta D, Sarma T K, Chowdhury D and Chattopadhyay A 2005 J. Colloid Interface Sci. 283153

[8] Drelinkiewicz A, Waksmundzka-Gora A, Sobczak J W and Stejskal J 2007 Appl. Catal. A: Gen. 333219

[9] Zhao C, Xing S, Yu Y, Zhang W and Wang C 2007 Microelectron. J. 38316

[10] Willner I, Willner B and Katz E 2007 Bioelectrochemistry 70 2

[11] Blinova N V, Stejskal J, Trchova M, Ciric-Marjanovic G and Sapurina I 2007 J. Phys. Chem. B 1112440

[12] Sun L J, Liu X X, Lau K K T, Chen L and Gu W M 2008 Electrochim. Acta. $\mathbf{5 3} 3036$

[13] Bessiere A, Duhamel C, Badot J C, Lucas V and Certiat M C 2004 Electrochim. Acta 492051

[14] Halvorson C, Cao Y, Moses D and Heeger A J 1993 Synth. Met. 573941

[15] Wang H L, MacDiarmid A G, Wang Y Z, Gebier D D and Epstein A J 1996 Synth. Met. 7833

[16] Kaneto K, Kaneko M, Min Y and MacDiarmid A G 1995 Synth. Met. 712211 
[17] Soto-Oviedo M A, Araujo O A, Faez R, Rezende M C and DePaoli M A 2006 Synth. Met. 1561249

[18] Kalendova A, Vesely D and Stejskal J 2008 Progr. Org. Coat. 62 105

[19] Stejskal J, Kratochv1l P and Jenkins A D 1996 Polymer 37367

[20] Christina O B, Xinwei H, Wyatt N and Richard B K 2017 Chem. Soc. Rev. 461510

[21] Ali E 2010 Nanostructured conductive polymers (Chichester, UK: John Wiley \& Sons Ltd.) p 19

[22] Hari Singh N 2001 Handbook of advanced electronic and photonic materials and devices (London, UK: Academic Press) $\mathrm{p} 1$

[23] Haines P J 1995 Thermal methods of analysis (London, UK: Springer Science)

[24] Shirsath N B, Gupta G R, Gite V V and Meshram J S 2017 Bull. Mater. Sci. 4163

[25] Zulkhairi Z, Nurul F A H, Mubaraq H V S, Shafiqul Islam A K M, Uda H and Ahmad M N 2015 J. Nanomater. 2015 218204
[26] Jiahua Z, Suying W, Lei Z, Yuanbing M, Jongeun R, Neel H et al 2011 J. Mater. Chem. 213952

[27] Arora M and Gupta S K 2008 ICOPVS

[28] Saini P, Arora M, Arya S K and Tawale J S 2014 Indian J. Pure Appl. Phys. 52175

[29] Agrawalla R K, Paul S, Sahoo P K, Chakraborty A K and Mitra A K 2015 J. Appl. Polym. Sci. 13241692

[30] Agrawalla R K, Meriga V, Paul R, Chakraborty A K and Mitra A K 2016 EXPRESS Polym. Lett. 9780

[31] Venkanna M, Sreeramulu V, Sivaprakash S, Caroline C and Dhanak V R 2015 J. Appl. Polym. Sci. 13242766

[32] Burns J A and Verall R E 1974 Thermochim. Acta. 9277

[33] Dortmund Data Bank. http://www.ddbst.com/en/EED/PCP/ HCP_C4577.php

[34] Preeti A T, Yadav S M and Gupta G R 2014 Polym. Bull. 71 1349

[35] Kumar D and Chandra R 2001 Indian J. Eng. Mater. Sci. 8 209

[36] Jiaxing H and Richard B K 2004 J. Am. Chem. Soc. 126851 